\title{
Autoimagem Genital, Função Sexual e Desconforto no Assoalho Pélvico em Mulheres Jovens Universitárias
}

\author{
Melissa Medeiros Braz, ${ }^{1}$ Emiláini Sacerdote Rister ${ }^{2}$
}

\section{RESUMO}

Mulheres com autoimagem genital negativa podem apresentar disfunções sexuais, as quais podem estar relacionadas a desconfortos no assoalho pélvico. O objetivo deste estudo é investigar a relação entre autoimagem genital, função sexual e desconforto no assoalho pélvico em mulheres jovens universitárias. Trata-se de um estudo observacional e transversal com 102 acadêmicas de Graduação e Pós-Graduação, de um total de 135 participantes, entre 18 e 29 anos, sexualmente ativas. Foram aplicados uma ficha de identificação relativa a dados pessoais, anamnese, queixas e atividade sexual, os questionários Female Sexual Function Index (FSFI), Pelvic Floor Distress Inventory (PFDI-SF-20) e Female Genital Self-Image Scale (FGSIS). O escore total do FGSIS indicou autoimagem genital positiva, FSFI boa função sexual e o PFDI-20 desconforto no assoalho pélvico baixo. Observou-se relação positiva entre autoimagem genital e função sexual e relação inversa com o desconforto no assoalho pélvico. As mulheres apresentaram autoimagem genital positiva, boa função sexual e baixo desconforto no assoalho pélvico, e as variáveis se relacionavam entre si.

Palavras-chave: Disfunções sexuais fisiológicas; autoimagem; diafragma da pelve.

GENITAL SELF-IMAGE, SEXUAL FUNCTION AND PELVIC FLOOR DISCOMFORT IN YOUNG UNIVERSITARY WOMEN

\section{ABSTRACT}

Women with negative genital self-image may experience sexual dysfunction. Still, it can be related to discomfort in the pelvic floor. The aim of this study is to investigate the relationship between genital self-image, sexual function and discomfort in the pelvic floor in young university women. This is an observational and cross-sectional study with 102 undergraduate and graduate students, from a total of 135 participants, between 18 and 29 years old, sexually active. An identification form relating to personal data, anamnesis, complaints and sexual activity, the Female Sexual Function Index (FSFI), Pelvic Floor Distress Inventory (PFDI-SF-20) and Female Genital Self-Image Scale (FGSIS) questionnaires were applied. The total FGSIS score indicated positive genital self-image, FSFI good sexual function and PFDI-20 discomfort in the low pelvic floor. There was a positive relationship between genital self-image and sexual function and an inverse relationship with discomfort in the pelvic floor. Women had positive genital self-image, good sexual function and low discomfort in the pelvic floor, and the variables were related to each other.

Keywords: Physiological sexual dysfunctions; self image; pelvis diaphragm.

RECEBIDO EM: 6/8/2020

MODIFICAÇÕES SOLICITADAS EM: 10/11/2020

ACEITO EM: 1\%/6/2021

\footnotetext{
${ }^{1}$ Autora correspondente. Universidade Federal de Santa Maria - UFSM. Av. Roraima, № 1000 Cidade Universitária, Bairro Camobi. Santa Maria/RS, Brasil. CEP 97105-900. http://lattes.cnpq. br/3515748001130422. https://orcid.org/0000-0002-9138-0656. melissabraz@hotmail.com

2 Universidade Federal de Santa Maria - UFSM. Santa Maria/RS, Brasil.
} 


\section{INTRODUÇÃO}

A autoimagem genital é um subcampo da pesquisa de imagem corporal que permite avaliar os sentimentos e crenças das mulheres sobre seus próprios genitais. ${ }^{1}$ Mulheres mais velhas, deprimidas e com baixa autoimagem genital tendem a apresentar pior função sexual. ${ }^{2}$ Por outro lado, mulheres que apresentam uma imagem positiva de seus órgãos genitais frequentemente têm sentimentos positivos sobre o corpo em geral, e reduzem as preocupações relacionadas ao seu corpo durante encontros sexuais. Essa imagem genital positiva pode ser relacionada a um aumento da satisfação sexual entre mulheres. ${ }^{3}$

Assim, a autoimagem que a mulher tem do seu corpo e da sua genitália interfere na saúde sexual. ${ }^{4}$ Esse assunto, no entanto, pode ser um tabu para muitas mulheres, ou um indicativo de constrangimento que possam sentir com relação à aparência de seus genitais. ${ }^{5}$

A função sexual é um componente integral da qualidade de vida e a autoimagem genital pode ser um bom indicador de possíveis Disfunções Sexuais (DS) em mulheres, especialmente aquelas com distúrbios que gerem desconforto no assoalho pélvico. ${ }^{2}$ Mulheres com alguma DS tendem a apresentar uma maior preocupação com a imagem corporal e percepção da genitália ${ }^{6}$ e as com função sexual adequada tendem a apresentar uma autoimagem genital mais positiva. $^{7}$

A autoimagem genital representa um componente potencialmente importante da saúde sexual feminina que tem sido sub-representado, tanto na literatura sobre sexualidade feminina quanto na prática clínica. Claramente, trata-se de um tópico que merece uma exploração mais aprofundada das perspectivas médicas e psicossociais, a fim de melhor elucidar sua relação com a experiência sexual feminina. ${ }^{8}$

A relação entre função sexual, autoimagem genital e o desconforto no assoalho pélvico em universitárias ainda é pouco investigada, necessitando de mais estudos para, a partir deles, auxiliar os profissionais da área da saúde nas tomadas de decisão sobre a abordagem e o tratamento de mulheres jovens.

Desta forma, a pesquisa propõe-se a responder: Existe relação entre autoimagem genital, função sexual e desconforto no assoalho pélvico em mulheres jovens universitárias?

\section{MATERIAIS E MÉTODOS}

Trata-se de uma pesquisa observacional com abordagem quantitativa e caráter transversal. O estudo foi realizado com 102 acadêmicas dos cursos de Graduação e Pós-Graduação de uma universidade pública do interior do Rio Grande do Sul, de um total de 135 participantes, jovens adultas com idades entre 18 e 29 anos e sexualmente ativas. Foram excluídas universitárias que tivessem dificuldade para responder ao questionário por fatores alo ou autopsíquicos, gestantes e puérperas (até o puerpério remoto) e também aquelas que mesmo após responder ao questionário não se incluíram nos critérios deste estudo.

Editora Unijuí - Revista Contexto \& Saúde - ISSN 2176-7114 - v. 21, n. 44, out./dez. 2021 


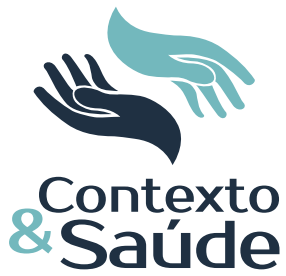

A coleta dos dados ocorreu dos meses de setembro de 2019 a março de 2020. A pesquisa foi realizada após aprovação do Comitê de Ética em Pesquisa (CEP) institucional sob o número de parecer 3.415.891. As universitárias foram abordadas no ambiente acadêmico durante os intervalos das aulas e informadas sobre o conteúdo da pesquisa e seu procedimento. Todas foram devidamente orientadas sobre os questionários a serem aplicados. Todos os instrumentos foram autorrespondidos.

Foram utilizados quatro instrumentos para a obtenção de dados, o primeiro uma ficha de identificação adaptada de Herbenick e Reece, ${ }^{9}$ composta por questões abertas e fechadas, relativas a dados pessoais, anamnese, queixas e atividade sexual. Em seguida foi aplicado o questionário Female Sexual Function Index (FSFI), validado e traduzido para o português, a fim de avaliar a função sexual. O FSFI é composto por 19 questões com 5 a 6 opções de resposta, com duas questões relacionadas ao desejo, quatro à excitação e lubrificação, três ao orgasmo, satisfação e a dor. A pontuação de cada dimensão varia entre 1,2 e 6 ou entre 0 e 6 , e a pontuação total do FSFI varia entre 2 e 36 , com pontuações altas indicando melhor função sexual. Foi adotado como ponto de corte do FSFI a pontuação de 26,55 , a fim de predizer disfunções sexuais para este grupo. Para a análise dos domínios foram utilizados os seguintes pontos de corte: Desejo: 4,28, Excitação: 5,08, Lubrificação: 5,45, Orgasmo: 5,05, Satisfação: 5,04 e Dor: 5,51, ${ }^{10}$

O Pelvic Floor Distress Inventory (PFDI-SF-20), validado e traduzido para o Brasil,,11 é composto por 20 questões divididas em 3 domínios (bexiga, intestino, pelve) em suas subescalas: Inventário de Distúrbio de Prolapso de Órgão Pélvico (POPDI-6), Inventário de Distúrbio Colorretal-Anal (CRADI-8) e Inventário de Distúrbio Urinário (UDI-6). Começa-se perguntando ao paciente se tem o sintoma descrito em cada item. Se a resposta for sim, existe um escore de classificação em que o paciente pode escolher a resposta, com um escore de subescala de $0 \mathrm{a}$ 100. Quanto maior a pontuação, maior é o desconforto dos sintomas de Disfunções do Assoalho Pélvico (DAP). ${ }^{11}$

O Female Genital Self-Image Scale (FGSIS) oferece um meio eficaz de avaliar a autoimagem genital. Tem 7 itens que avaliam os sentimentos e crenças das mulheres sobre seus próprios genitais, usando uma escala de resposta de 4 pontos (concordo totalmente, concordo, discordo, discordo totalmente). As pontuações dos entrevistados em cada item são somadas para uma pontuação total, variando de 7 a 28, com pontuações mais altas indicando uma autoimagem genital mais positiva. ${ }^{1}$

As variáveis categóricas foram analisadas descritivamente por meio de frequência simples e porcentagens e as numéricas por medidas de posição e dispersão. Foi utilizado o teste $U$ de Mann Whitney para comparar a autoimagem genital entre os domínios do FSFI categorizados conforme a ocorrência de DS. O teste de correlação de Spearman foi utilizado para correlacionar a autoimagem genital com os domínios do FSFI. A classificação quanto à força da correlação seguiu os seguintes critérios: rho $<0,30$ como baixa correlação; $0,30 \leq$ rho $\leq 0,60$ como moderada correlação e rho $>0,60$ como alta correlação. O tamanho de efeito (ES) das comparações significativamente diferentes foi determinado pela fórmula ES = $z / V N$ : $\leq 0,10$ - efeito nulo; 0,11 a 0,29 - efeito fraco; 0,30 a 0,49 - efeito modera-

Editora Unijuí - Revista Contexto \& Saúde - ISSN 2176-7114 - v. 21, n. 44, out./dez. 2021 
do e $\geq 0,50$ - efeito grande. Para medida de efeito da correlação foi empregado o Coeficiente de Determinação $\left(R^{2}\right)$. Foi utilizado nível de significância de $5 \%$ para todos os testes. As análises estatísticas foram realizadas no programa SPSS 22.0.

\section{RESULTADOS}

Foram respondidos 135 questionários, sendo analisados pela elegibilidade 102. As participantes tinham média de idade $22,05 \pm 2,42$ anos

Figura 1 - Fluxograma dos critérios de elegibilidade

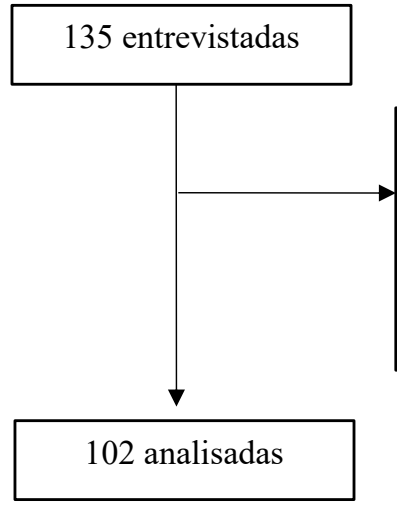

Fonte: Elaborada pelos autores.

A Tabela 1 apresenta as características sociodemográficas, ginecológicas e clínicas das participantes do estudo, das quais $78,22 \%$ declararam-se de cor branca, 69,61\% com companheiro(a) e 80,40\% heterossexuais. Quanto aos aspectos de saúde ginecológica, 63,72\% haviam realizado uma consulta ginecológica no último ano e $13,72 \%$ nunca realizaram nenhum tipo de consulta ao ginecologista. Além disso, 69,61\% das mulheres fazem uso de anticoncepcional e $13,73 \%$ de antidepressivo.

Tabela 1 - Características sociodemográficas, ginecológicas e clínicas das mulheres jovens universitárias

\begin{tabular}{lc}
\hline Variáveis & $\mathbf{n}(\%)$ \\
\hline Cor da pele $^{*}$ & \\
Branca & $79(78,22)$ \\
Parda & $15(14,85)$ \\
Preta & $7(6,93)$ \\
\hline Estado civil & \\
Com companheiro(a) & $71(69,61)$ \\
Sem companheiro(a) & $31(30,39)$ \\
\hline Orientação sexual & \\
Heterossexual & $82(80,40)$ \\
Homossexual & $15(14,70)$ \\
Bissexual & $5(4,90)$ \\
\hline Uso de antidepressivo & \\
Não & $88(86,27)$ \\
Sim & $14(13,73)$ \\
\hline
\end{tabular}




\begin{tabular}{lc}
\hline Uso de pílula anticoncepcional & $71(69,61)$ \\
Sim & $31(30,39)$ \\
Não & \\
\hline Frequência de consulta ginecológica & $14(13,72)$ \\
Nenhuma no ano & $65(63,72)$ \\
Uma vez por ano & $23(22,55)$ \\
Duas vezes ou mais por ano & \\
\hline Realiza teste preventivo & $63(61,76)$ \\
Sim & $39(38,24)$ \\
Não & \\
\hline Realizou cirurgia ginecológica & $97(95,10)$ \\
Não & $5(4,90)$ \\
Sim & $66(64,70)$ \\
\hline Possui sintomas de IU & $36(35,30)$ \\
Não & $65(63,72)$ \\
Sim & $37(36,28)$ \\
\hline Possui sintomas de IF & \\
Não & $62(60,78)$ \\
Sim & $40(39,22)$ \\
\hline Possui sintomas de POP & \\
Sim & \\
Não & \\
*: Variável que contém dado missing. ${ }^{*} p \leq 0,05$. & \\
$\quad$ Fontaborada pelos autores. &
\end{tabular}

A Tabela 2 apresenta a avaliação da autoimagem genital por meio do FGSIS e da função sexual pelo FSFI. Observa-se que as participantes avaliavam sua autoimagem genital de forma positiva, com mediana de 24 (7) pontos no escore total do FGSIS. O escore total do FSFI indica que estas mulheres não apresentam disfunção sexual, porém os domínios desejo, orgasmo e lubrificação foram afetados. As médias dos escores do PFDI-20 indicam baixo desconforto no assoalho pélvico em todos os domínios.

Tabela 2 - Representação dos domínios e do escore total do FGSIS, FSFI e PFDI-20 em mulheres jovens universitárias, apresentados em mediana e intervalo interquartil

\begin{tabular}{lc}
\hline FGSIS & Mediana (IIQ) \\
\hline Segurança positiva sobre os genitais & $3(1)$ \\
Satisfação com a aparência dos genitais & $3(1)$ \\
Conforto em deixar um parceiro(a) sexual olhar os genitais & $3(1)$ \\
Bom odor dos genitais & $3(1)$ \\
Funcionamento dos genitais & $4(1)$ \\
Conforto em deixar um profissional da saúde examinar os genitais & $3(1)$ \\
Vergonha dos genitais & $3,50(1)$ \\
Pontuação total (FGSIS) & $24(7)$ \\
& \\
FSFI & \\
\hline Desejo sexual & $3,60(1,20)$ \\
Excitação sexual & $5,10(1,20)$
\end{tabular}




\begin{tabular}{lc} 
Lubrificação vaginal & $5,40(1,20)$ \\
Orgasmo & $4,80(2,00)$ \\
Satisfação sexual & $5,20(1,60)$ \\
Dor sexual & $5,20(1,60)$ \\
Pontuação total (FSFI) & $28,36(4,82)$ \\
PFDI-20 & $4,17(8,33)$ \\
\hline${ }^{*}$ POPDI-6 & $6,25(7,03)$ \\
${ }^{\text {}}$ CRADI-8 & $4,17(8,33)$ \\
\&UDI-6 & $16,67(19,79)$ \\
Pontuação total (PFDI-20)
\end{tabular}

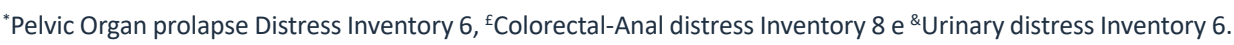
Fonte: Elaborada pelos autores.

A Tabela 3 apresenta a comparação da autoimagem genital e função sexual por meio do FSFI. Observou-se que, em mediana, as mulheres sem DS apresentaram valores mais positivos da autoimagem genital na função sexual geral, excitação sexual, lubrificação vaginal, orgasmo, satisfação sexual e dispareunia, se comparadas às mulheres com DS. O tamanho de efeito das comparações significativas entre a autoimagem genital e a função sexual foi considerado moderado para a função sexual geral, orgasmo e dispareunia, e fraco para excitação sexual, lubrificação vaginal e satisfação sexual.

Tabela 3 - Comparação da autoimagem genital com os domínios do FSFI entre mulheres universitárias com e sem DS ( $n=102)$

\begin{tabular}{|c|c|c|c|c|}
\hline \multirow[b]{2}{*}{ Variáveis } & \multirow[b]{2}{*}{ n (\%) } & \multicolumn{3}{|c|}{ Autoimagem genital } \\
\hline & & Mediana (IIQ) & $\mathbf{P}$ & ES \\
\hline Função sexual geral & & & $0,001^{*}$ & 0,326 \\
\hline Sem DS & $72(70,60)$ & $25(6)$ & & \\
\hline Com DS & $30(29,40)$ & $21,50(6)$ & & \\
\hline Desejo sexual & & & 0,110 & - \\
\hline Com DS & $97(95,10)$ & $24(7)$ & & \\
\hline Sem DS & $5(4,90)$ & $27(4)$ & & \\
\hline Excitação sexual & & & $0,005^{*}$ & 0,275 \\
\hline Sem DS & $54(52,90)$ & $25,50(6)$ & & \\
\hline Com DS & $48(47,10)$ & $22(7)$ & & \\
\hline Lubrificação vaginal & & & $0,036^{*}$ & 0,207 \\
\hline Com DS & $63(61,80)$ & $22(6)$ & & \\
\hline Sem DS & $39(38,20)$ & $26(6)$ & & \\
\hline Orgasmo & & & $0,001^{*}$ & 0,329 \\
\hline Com DS & $58(56,90)$ & $22(7)$ & & \\
\hline Sem DS & $44(43,10)$ & $26(6)$ & & \\
\hline Satisfação sexual & & & $0,017^{*}$ & 0,237 \\
\hline Sem DS & $65(63,70)$ & $25(7)$ & & \\
\hline Com DS & $37(36,30)$ & $22(6)$ & & \\
\hline Dispareunia & & & $<0,001^{*}$ & 0,368 \\
\hline Com DS & $60(58,80)$ & $22(7)$ & & \\
\hline Sem DS & $42(41,20)$ & $26(5)$ & & \\
\hline
\end{tabular}

IIQ: Intervalo Interquartil. ES: Tamanho de Efeito. DS: Disfunção Sexual. * ${ }^{*} \leq 0,05$.

Fonte: Elaborada pelos autores. 
A Tabela 4 apresenta a correlação da autoimagem genital com o FSFI e PFDI-20.

Observa-se uma correlação fraca entre a autoimagem genital com o desejo, lubrificação e satisfação, e moderada com a excitação, orgasmo, dor e o escore total do FSFI. Ao analisar o coeficiente de determinação observou-se que $6,20 \%$ da variação da autoimagem genital pode ser explicada pelo desejo sexual, 10,04\% pela excitação sexual, 5,90\% pela lubrificação vaginal, 9,61\% pelo orgasmo, $8,70 \%$ pela satisfação sexual, $17,47 \%$ pela dor sexual e $20,97 \%$ pela pontuação total no FSFI.

Observa-se também uma correlação inversa fraca entre a autoimagem genital com POPDI-6, CRADI-8, UDI-6 e o escore total do PFDI. Analisando o coeficiente de determinação, 9,48\% da variação da autoimagem genital pode ser explicada pelo POPDI-6, 5,29\% pelo CRADI-8, 4,12\% pelo UDI-6 e 12,81\% pela pontuação total no PFDI-20.

Tabela 4 - Correlação da autoimagem genital com o FSFI e PFDI-20 em mulheres jovens universitárias

\begin{tabular}{lccc}
\hline & \multicolumn{3}{c}{ Autoimagem genital } \\
\hline FSFI & Rho & $\mathbf{P}$ & $\mathbf{R}^{\mathbf{2}}$ \\
Desejo sexual & 0,249 & $0,012^{*}$ & 0,0620 \\
& & & \\
Excitação sexual & 0,317 & $0,001^{*}$ & 0,1004 \\
Lubrificação vaginal & 0,243 & $0,014^{*}$ & 0,0590 \\
Orgasmo & 0,310 & $0,002^{*}$ & 0,0961 \\
Satisfação sexual & 0,295 & $0,003^{*}$ & 0,0870 \\
Dor & 0,418 & $<0,001^{*}$ & 0,1747 \\
Pontuação total & 0,458 & $<0,001^{*}$ & 0,2097
\end{tabular}

PFDI-20

\begin{tabular}{lccc}
\hline POPDI-6 & $-0,308$ & $0,002^{*}$ & 0,0948 \\
CRADI-8 & $-0,230$ & $0,020^{*}$ & 0,0529 \\
UDI-6 & $-0,203$ & $0,041^{*}$ & 0,0412 \\
PFDI-20 total & $-0,358$ & $<0,001^{*}$ & 0,1281 \\
\hline
\end{tabular}

FSFI: Female Sexual Function Index; PFDI-20: Pelvic Floor Distress Inventory; rho: coeficiente de correlação; $R^{2}$ : coeficiente de determinação; ${ }^{*} p \leq 0,05$.

Fonte: Elaborada pelos autores.

\section{DISCUSSÃO}

O presente estudo explorou a relação entre autoimagem genital, função sexual e desconforto no assoalho pélvico de 102 mulheres jovens. Observou relação positiva entre autoimagem genital e função sexual, com todos os domínios do FSFI. Da mesma forma, constatou-se relação inversa fraca entre autoimagem genital e desconforto no assoalho pélvico, por meio dos domínios POPDI-6, CRADI-8, UDI-6 e do escore total do PFDI. 
Em uma pesquisa realizada nos Estados Unidos da América utilizando o FGSIS, as participantes relataram uma visão relativamente positiva de seus órgãos genitais, com aproximadamente $75 \%$ da amostra relatando que "concordam" ou "concordam fortemente" com cada uma das declarações $(21,31 \pm 4,31) .{ }^{1}$ Outro estudo utilizando o FGSIS, com mulheres jovens (média de idade de 23,20 anos), apresentou média de pontuação de 21,92, também indicando uma autoimagem genital positiva. ${ }^{3} \mathrm{O}$ nosso estudo apresentou no FGSIS uma média de 24 pontos, demonstrando que as mulheres apresentam autoimagem genital positiva.

No estudo de Zheng, ${ }^{12}$ a autoimagem genital negativa foi associada a sobrepeso, obesidade, convivência, não estar casado, casamento, amamentação e uso de medicamentos psicotrópicos. De acordo com Goldsmith et al., ${ }^{13}$ mensagens positivas dos parceiros(as) aumentam a autoestima e confiança das mulheres em relação à sua aparência, e/ou aumentam sua própria apreciação e aceitação de seus corpos.

As escalas de autoimagem genital oferecem um meio eficaz de avaliar os sentimentos das mulheres sobre seus órgãos genitais. As informações que as escalas fornecem parecem se relacionar com a quantidade de depressão, angústia sexual e desejo que podem estar ocorrendo com essas mulheres. ${ }^{14}$

No estudo de Komarnicky et al. ${ }^{3}$ com mulheres na média de 23,20 anos, a autoimagem genital foi significativamente relacionada aos domínios do FSFI, que observou que a autoimagem genital mais positiva foi associada a escores reduzidos de disfunção sexual. Assim, manter atitudes negativas sobre os órgãos genitais pode levar à diminuição no desejo e na excitação e pode potencialmente afetar o orgasmo e a função sexual de forma geral.

Nosso estudo com 102 jovens apresentou uma média de escore total de 28,36 pontos no FSFI, indicando boa função sexual. Os domínios que apresentaram alteração foram o desejo, orgasmo e lubrificação. Estudo avaliou a função sexual de cem universitárias com idades entre 18 e 35 anos em São Paulo por meio do FSFI. O escore geral das estudantes foi de 28,30 pontos, significando boa satisfação sexual, no entanto $28 \%$ das mulheres apresentaram escores indicativos para DS. Os domínios que foram identificados como mais acometidos foram o desejo e a excitação, o que consequentemente leva à dificuldade para chegar ao orgasmo. ${ }^{15}$

Estudo ${ }^{16}$ que analisou 149 estudantes da área da saúde com média de idade de 21 anos, observou que $28,8 \%$ das mulheres apresentavam predição para disfunção sexual em todos os domínios.

$\mathrm{Na}$ sociedade atual estão muito presentes relações afetivas mais breves, com menos compromisso e intimidade com o parceiro. Tais fatores podem estar influenciando negativamente a função sexual nessa população. Entre as mulheres jovens os fatores socioculturais podem estar influenciando mais do que os fatores biológicos. ${ }^{17}$ Para Zheng et al., ${ }^{12}$ o bem-estar sexual é influenciado por múltiplos relacionamentos, fatores socioculturais, psicológicos e biológicos. Fatores como ansiedade e depressão aumentaram também o risco de DS, e vice-versa. ${ }^{18}$ Mulheres com transtorno de ansiedade apresentam um aumento da insatisfação 
sexual, afetando negativamente a função sexual e a qualidade de vida (QV) e expressando piora significativa no desejo sexual, excitação e níveis de orgasmo. ${ }^{19}$

Observou-se em nosso estudo que as mulheres apresentavam baixo desconforto no assoalho pélvico em todos os domínios. Um estudo dividiu as muIheres em grupo jovem (média 28,6 anos) e grupo mais velho (média 61,8 anos) e avaliou o desconforto no assoalho pélvico pelo PFDI-20. Constatou-se que a idade foi correlacionada positivamente com os escores de PFDI-20 e os escores de UDI-6. ${ }^{20}$

Disfunções do Assoalho Pélvico (DAP), como prolapsos, Incontinência Urinária (IU) e Incontinência Fecal (IF) podem estar associadas a sintomas dolorosos ou desconfortos, ${ }^{21}$ que podem ter um impacto na QV das mulheres. A utilização do questionário de desconforto no assoalho pélvico (PFDI-20) ajuda a avaliar esses distúrbios, contribuindo para a melhoria dos cuidados de saúde nessas muIheres. ${ }^{11}$

Em um estudo realizado com 755 mulheres com média de idade de 56 anos que utilizou o PFDI os autores observaram que as disfunções do assoalho pélvico, bem como o desconforto, estiveram relacionados a disfunções sexuais, como baixo desejo e níveis moderados de excitação e orgasmo. ${ }^{22}$

A DS em mulheres que sofrem de disfunções e desconforto no assoalho pélvico está fortemente relacionada à imagem corporal, visto que a disposição de se envolver em atividades sexuais é afetada e outros efeitos adversos também são observados na excitação, orgasmo e dispareunia em mulheres com DAP e desconforto. ${ }^{23} \mathrm{O}$ diagnóstico precoce da DSF em mulheres jovens, juntamente com a intervenção intersdisciplinar, pode contribuir para a melhoria da prática sexual e da QV. ${ }^{16}$

\section{CONCLUSÃO}

As mulheres deste estudo apresentaram autoimagem genital positiva e boa função sexual. Observou-se que a autoimagem genital positiva está relacionada à melhor função sexual, excitação, lubrificação, orgasmo, satisfação sexual e dispareunia. Também foi observado baixo desconforto no assoalho pélvico, com relação entre a autoimagem genital e o desconforto no assoalho pélvico.

As limitações encontradas durante a pesquisa foram a amostra por conveniência, a disponibilidade de horários das estudantes, os questionários extensos e o próprio tema que ainda se configura como um tabu, por sentirem-se desconfortáveis em relação a ele. Também devido à pandemia da Covid-19 ficou impossibilitada a coleta de dados por um tempo maior.

\section{REFERÊNCIAS}

${ }^{1}$ Herbenick D, Schick V, Reece M, Sanders S, Dodge B, Fortenberry JD. The Female Genital Self-Image Scale (FGSIS): results from a nationally representative probability sample of women in the United States. J Sex Med. 2011 jan.;8(1):158-66. 
${ }^{2}$ Handelzalts JE, Yaakobi T, Levy S, Peled Y, Wiznitzer A, Krissi H. The impact of genital self-image on sexual function in women with pelvic floor disorders. Eur J Obstet Gynecol Reprod Biol. 2017 apr.;211:164-8.

${ }^{3}$ Komarnicky T, Skakoon-Sparling S, Milhausen RR, Breuer R. Genital Self-Image: Associations with Other Domains of Body Image and Sexual Response. J Sex Marital Ther. 2019;45(6):524-37.

${ }^{4}$ Gomes et al. T. Imagem corporal e imagem genital feminina. Revista Científica Esc da Saúde. 2015;37-42.

${ }^{5}$ Laan E, Martoredjo DK, Hesselink S, Snijders N, van Lunsen RHW. Young women's genital self-image and effects of exposure to pictures of natural vulvas. J Psychosom Obstet Gynaecol. 2017 dez.;38(4):249-55.

${ }^{6}$ Ribeiro RTSK. Associação da imagem corporal e autoimagem genital com a função sexual de mulheres adultas jovens: estudo transversal. Escola Baiana de Medicina e Saúde Pública; 2019.

${ }^{7}$ Gillen MM, Markey $\mathrm{CH}$. A review of research linking body image and sexual well-being. Body Image. 2019 dezc.;31:294-301.

${ }^{8}$ Fudge MC, Byers ES. An exploration of psychosocial factors associated with female genital self-image. Vol. 37, Gender Issues. Byers, E. Sandra: Department of Psychology, University of New Brunswick, P. O. Box 4400, Fredericton, NB, Canada, E3B 5A3, byers@ unb.ca: Springer; 2020. p. 153-72.

${ }^{9}$ Herbenick D, Reece M. Development and validation of the female genital self-image scale. J Sex Med. 2010 maio;7(5):1822-30.

${ }^{10}$ Marques MG, Braz MM. Efeito do método pilates sobre a função sexual feminina. Fisioter Bras. 2017;18(1):63-8.

${ }^{11}$ Arouca MAF, Duarte TB, Lott DAM, Magnani PS, Nogueira AA, Rosa-E-Silva JC, et al. Validation and cultural translation for Brazilian Portuguese version of the Pelvic Floor Impact Questionnaire (PFIQ-7) and Pelvic Floor Distress Inventory (PFDI-20). Int Urogynecol J. 2016 jul;27(7):1097-106.

12 Zheng J, Skiba MA, Bell RJ, Islam RM, Davis SR. The prevalence of sexual dysfunctions and sexually related distress in young women: a cross-sectional survey. Fertil Steril. 2020 fev.;113(2):426-34.

${ }^{13}$ Goldsmith KM, Byers ES. Perceived impact of body feedback from romantic partners on young adults' body image and sexual well-being. Vol. 17, Body Image. Goldsmith, Kaitlyn M.: Department of Psychology, University of New Brunswick, P.O. Box 4400, Fredericton, NB, Canada, E3B 5A3, kaitlyn.goldsmith@unb.ca: Elsevier Science; 2016. p. 161-70.

${ }^{14}$ Braz MM. Autoimagem genital feminina no processo de envelhecimento: revisão integrativa de literatura. 2019;22:189-207.

${ }^{15}$ Damasceno SDO. Avaliação da satisfação sexual em universitárias. 2019;11(1):1-6.

${ }^{16}$ I JTS, Rodrigues T, Pereira C, Chaves M, lii A, Federal U, et al. Self-reported assessment of female sexual function among Brazilian undergraduate healthcare students: a cross-sectional study ( survey ). 2018;136(4):333-8.

${ }^{17}$ Arcanjo S, Karbage L, I DMS, Oliveira M, Oriá B, Federal U. Sexual function of undergraduate women: a comparative study between Brazil and Italy. 2018;71(suppl 3):1428-34.

${ }^{18}$ Bianchini P, Paula S, Navarro A, Rita M, Lúcia L, Lara S. Sexual Function of Women with Infertility Função sexual de mulheres com infertilidade. 2018.

${ }^{19}$ Faculty M. Evaluation of body image, sexual dysfunctions and quality of life in female patients with generalized anxiety disorder. 2018;1-4.

${ }^{20}$ Alshiek J, Wei Q, Jalalizadeh M, Chitnis P, Shobeiri SA. The Effect of Aging on Pelvic Floor Pressure Measurements in Nulliparous Women. 2020;751-69.

${ }^{21}$ Bo K, Frawley HC, Haylen BT, Abramov Y, Almeida FG, Berghmans B, et al. An International Urogynecological Association (IUGA)/International Continence Society (ICS) joint report on the terminology for the conservative and nonpharmacological management of female pelvic floor dysfunction. Neurourol Urodyn. 2017 fev.;36(2):221-44. 
22 Li-Yun-Fong RJ, Larouche M, Hyakutake M, Koenig N, Lovatt C, Geoffrion R, et al. Is Pelvic Floor Dysfunction an Independent Threat to Sexual Function? A Cross-Sectional Study in Women With Pelvic Floor Dysfunction. J Sex Med. 2017 fev.;14(2):226-37.

${ }^{23}$ Roos A-M, Thakar R, Sultan AH, Burger CW, Paulus ATG. Pelvic floor dysfunction: women's sexual concerns unraveled. J Sex Med. 2014 mar.;11(3):743-52.

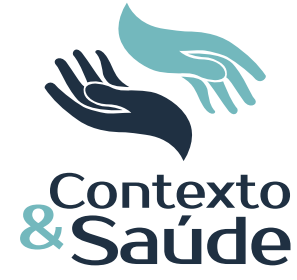

